\title{
Extreme ultraviolet tomography of multi-jet gas puff target for high-order harmonic generation
}

\author{
P. W. Wachulak $\cdot$ L. Węgrzyński $\cdot$ Z. Zápražný • \\ A. Bartnik $\cdot$ T. Fok $\cdot$ R. Jarocki $\cdot$ J. Kostecki $\cdot$ \\ M. Szczurek · D. Korytár $\cdot$ H. Fiedorowicz
}

Received: 17 October 2013/Accepted: 28 March 2014/Published online: 18 April 2014

(C) The Author(s) 2014. This article is published with open access at Springerlink.com

\begin{abstract}
A volume, tomographic reconstruction of a novel, multi-jet gas puff target, developed for possible applications in high-order harmonic generation (HHG), is presented. The target, produced by pulsed injection of argon gas through nozzle in a form of linearly oriented small orifices, has been characterized in the extreme ultraviolet at $13.5 \mathrm{~nm}$ wavelength. Target density estimations were performed, and 3-D representation of pulsed gaseous target has been obtained by combining 2-D shadowgram images, recorded at various rotation angles. More detailed information about higher-order jets, formed as a result of collisions of primary jets, was obtained. Tomographic studies of such type of targets dedicated for HHG have been obtained to our knowledge for the first time.
\end{abstract}

Electronic supplementary material The online version of this article (doi:10.1007/s00340-014-5829-7) contains supplementary material, which is available to authorized users.

\section{Introduction}

The characterization measurements of pulsed gas jets, gas puff targets, array of gas jets and dual-gas multi-jet targets proved to be useful in efficient generation of extreme ultraviolet (EUV) and soft X-ray (SXR) radiation $[1,2]$ and was already reported in a variety of papers, [3-10], using different diagnostic methods. The most common method that was used in the characterization measurements of various target systems is laser interferometry [11, 12]. Other studies show the possibility to perform characterization of rapidly evolving (in the nanosecond time scale) gaseous targets using shadowgraphy techniques [13-15]. In this study, however, to extract even more complete information about those targets, we combined radiography technique (shadowgraphy) [16], with tomography, which is capable of rendering 3-D objects from multiple projections.

Detailed characterization of target density is essential for quasi-phase-matching (QPM) in high-order harmonic generation (HHG) employing modulated density media and may strongly increase energy conversion efficiency [17-

\footnotetext{
J. Kostecki

e-mail: jkostecki@wat.edu.pl

M. Szczurek

e-mail: mszczurek@wat.edu.pl

H. Fiedorowicz

e-mail: hfiedorowicz@wat.edu.pl

Z. Zápražný · D. Korytár

Institute of Electrical Engineering, Slovak Academy of Sciences,

Dúbravská cesta 9,84104 Bratislava, Slovakia

e-mail: zdenko.zaprazny@savba.sk

D. Korytár

e-mail: dusan.korytar@savba.sk
} 
20]. QPM has been proven experimentally using gas-filled capillaries [21], gas-filled hollow-core fibers with a modulated inner diameter [22], coherent superposition of harmonics generated in two successive sources by the same laser pulse [23], however, with limited or no characterization measurements of gas density profiles in the laser interaction region, which are essential to perform numerical simulations and to understand the QPM process.

In this paper, we present application of EUV tomography to combine 2-D shadowgram projections into a volume reconstruction of multi-jet modulated density gas puff target for more precise study of the target density. This target, developed in our group, might have potential application for phase-matching in HHG experiments. 2-D projections alone are often insufficient for in-depth target characterization, especially if accurate density profiles are required. Tomographic method using hard X-ray energy range was already proven for nondestructive observing of the internal structure of selected objects across a wide range of scientific [24], industrial [25] and especially medical applications [26], for imaging biological tissues $[27,28]$. The tissues belong to the group of materials with low atomic number and low density, which is very similar to the gas objects [29]. These samples usually exhibit very low absorption contrast in the hard X-ray energy range [30,31]. Therefore, the selection of appropriate energy range (wavelength) considering the absorption of a sample is very important. Heavier elements with a higher atomic number require a shorter wavelength, or vice versa. For our gas target, the EUV backlighting method at $13.5 \mathrm{~nm}$ wavelength was used, based on the source described in detail in [32]. The target was formed by pulsed injection of gas through nozzle in a form of small orifices drilled in in-line geometry. Pulsed operation mode allows for sufficient removal of gas from the chamber and operation at $\sim 10^{-3}$ to $10^{-4}$ mbar chamber pressure during the valve operation, limiting EUV reabsorption in the neutral, residual gas. This method allows for imaging of target density features, unseen previously directly from EUV shadowgrams, which can now be studied in detail. The results of the studies will be useful for research on HHG and for understanding of the HHG process. Moreover, this method can be immediately applied to characterize targets used in experiments related to e-beam acceleration, ion acceleration, generation of EUV and SXR radiation, laser-matter interaction and EUV and SXR lasers.

\section{Experimental arrangement for EUV tomography}

The gas target is backlighted with EUV pulses at $13.5 \mathrm{~nm}$ wavelength at various angles, rendering 2-D projections of the object, and later, by combining those projections, we were able to obtain 3-D rendering of operating gas puff target.
The EUV pulses have been obtained by spectral selection of a xenon plasma emission. The spectral narrowing was performed using thin-film filters and a $\mathrm{Mo} / \mathrm{Si}$ multilayer mirror. Similar technique has been previously applied for characterization of gas jet xenon targets [33], elongated geometry gas targets [15] and multi-jet gas puff targets [13].

The experimental setup for EUV shadowgraphy, described in details elsewhere [14], is depicted in Fig. 1a. Pulses of EUV radiation are produced using a compact laser plasma EUV source based on a double-stream Xe/He gas puff target. More details about source optimization can be found in [34]. Emission at central wavelength $\lambda=13.5 \mathrm{~nm}$ with the bandwidth of about $1 \mathrm{~nm}$ was obtained using a flat $\mathrm{Mo} / \mathrm{Si}$ multilayer mirror, positioned at $45^{\circ}$ incidence angle for its theoretical peak reflectivity at $\lambda=13.5 \mathrm{~nm}$ of about $38 \%$ (measured value $\sim 31 \%$ ). Additionally to eliminate longer wavelengths from Xe plasma, including the visible light, a composite $\mathrm{Zr} / \mathrm{Si}_{3} \mathrm{~N}_{4}$ filter was used. The thickness of each layer was $200 \mathrm{~nm}$. Spectral measurements were performed using a transmission grating spectrometer (TGS), equipped with a $4 \mu \mathrm{m}$ period, free-standing grating located $720 \mathrm{~mm}$ from the plasma. An entrance slit, $33 \mu \mathrm{m}$ in width, was positioned $\sim 4 \mathrm{~mm}$ from the grating. A normalized Xe plasma emission spectrum, transmission curves of thin-film filters, the reflectivity curve of the $\mathrm{Mo} / \mathrm{Si}$ mirror and the EUV spectrum after spectral filtration are depicted in Fig. 2. Spectrally narrowed, quasi-monochromatic, pulsed EUV radiation with inverse relative bandwidth of $\lambda / \Delta \lambda=13.5$ illuminates gaseous target produced by electromagnetic valve system.

The gaseous target was formed by nozzles, supplied with gas pressurized inside gas reservoirs. The flow of the gas is controlled by a fast-acting electromagnetic valve. The valve can operate with gas backing pressures up to 5 bars. The nozzles form a multi-jet gas puff target by a repeatable and periodic modulation of gaseous target density. To facilitate the use of this kind of target in HHG experiments, we used argon gas.

Previously, as reported in [14], this target was preliminarily characterized and it was found that the target density profiles depend on a few parameters, such as the nozzle geometry, backing pressures of gas or gasses, supplied to the valve, the time duration of gas flow and finally on the mode of operation. The information on the target density profiles is very important for source development-related research (including HHG); however, obtaining twodimensional density maps and later, density profiles, does not allow to obtain a complete, 3-D picture of the investigated object (target). Thus, in this study, we employed a tomography to visualize in 3-D at the lithographic wavelength of $13.5 \mathrm{~nm}$, a rapidly changing phenomenon of a gaseous target formation. For that, we use arrangement of 

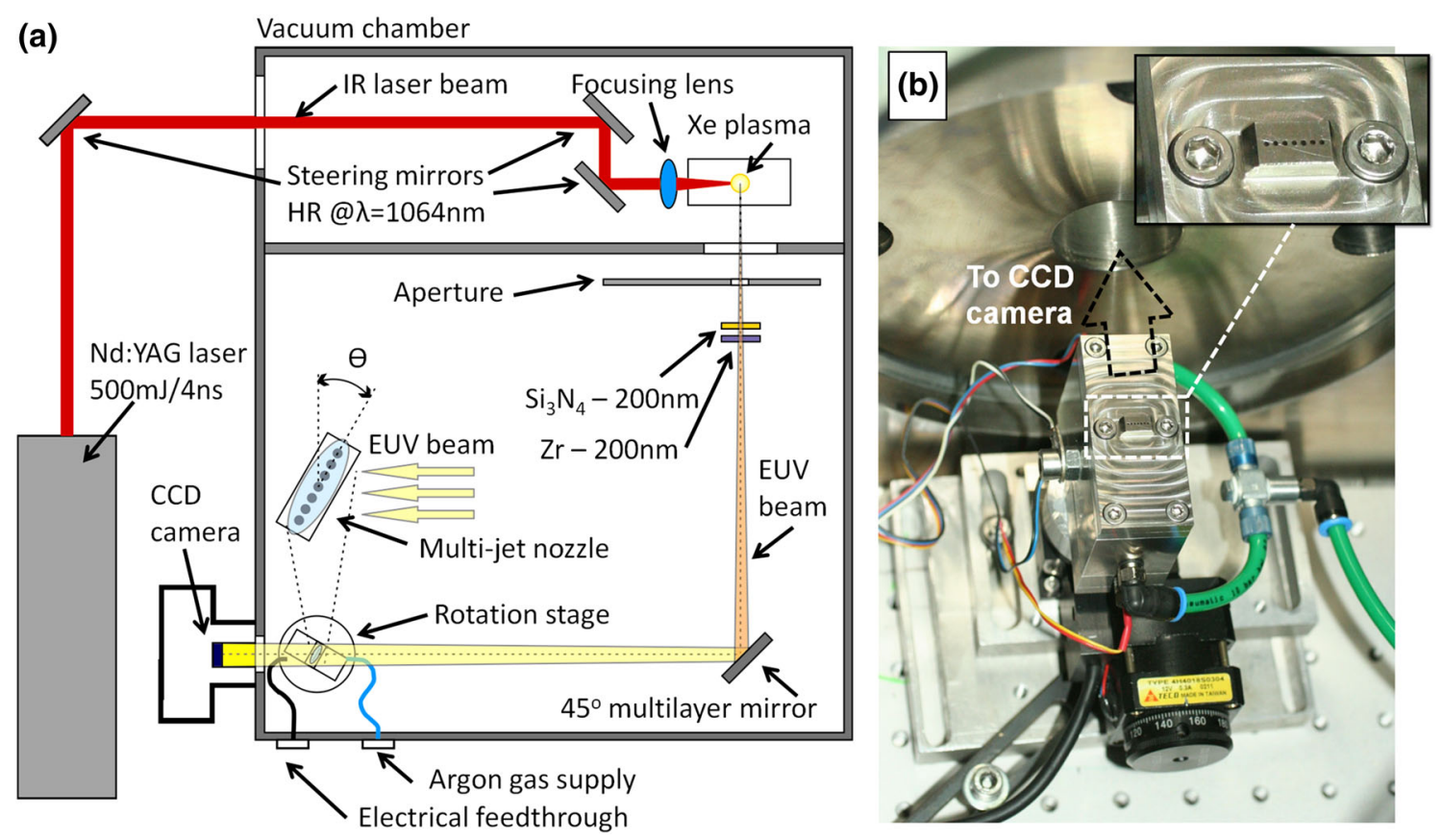

Fig. 1 Experimental arrangement for EUV tomography setup of a multi-jet modulated gas puff target. (a) Photographs of the valve (b) and the nozzles-small inset in the image. Argon was supplied to all seven nozzles

Fig. 2 Spectra obtained using TGS showing the EUV emission from Xe plasma, transmission curves for all filters, reflectivity curve for the $\mathrm{Mo} / \mathrm{Si}$ mirror and the EUV spectrum of radiation used in the tomography experiment after spectral narrowing. Transmission/ reflection curves are calculated based on data available from [37]

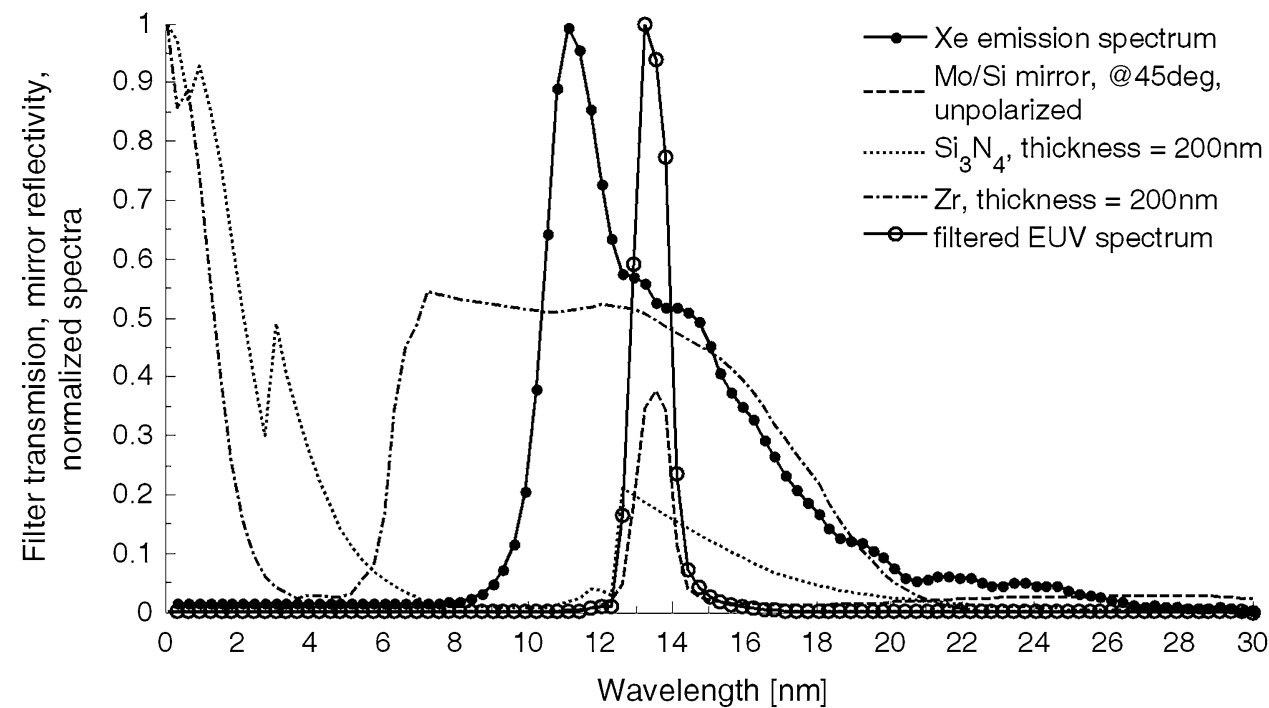

EUV light illuminating the target, locally and partially absorbed by the gas, forming an intensity image at the detector plane, further downstream the EUV beam. For each angle $\Theta$, depicted schematically in Fig. 1a, the shadowgrams of the multi-jet gas puff target were registered using a back-illuminated charge-coupled device (CCD) camera, X-vision M-25 (from Reflex company, Czech Republic), equipped with $512 \times 512$ pixels CCD chip, $0.5 \times 0.5 \mathrm{in}^{2}$. in size. The distances between the source to the characterized target and the source to the CCD camera were 880 and $995 \mathrm{~mm}$, respectively. Geometrical magnification of our system is equal to $1.13 \times$. 
Assuming EUV source size $\sim 200 \mu \mathrm{m}$, measured previously using pinhole camera, the spatial resolution possible to achieve in this configuration is approximately $30 \mu \mathrm{m}$, which corresponds roughly to a size of a single CCD pixel $(\sim 25 \mu \mathrm{m})$. This was confirmed experimentally by measuring resolution using a knife-edge criterion in shadowgrams at the edge of the valve, which results in a value of 1.5 pixels or $\sim 38 \mu \mathrm{m}$. The resolution might be further improved by moving the object closer to the detector; however, the ultimate resolution limit is the CCD pixel size. To ensure a proper pixel segmentation number of equiangular projections $N_{\mathrm{p}}$ has to fulfill the condition (1):

$N_{\mathrm{p}} \geq \frac{\pi}{2} \cdot N_{\mathrm{d}}$

where $N_{\mathrm{d}}$ is a number of detector pixels in the plane of object rotation. The angular step $\Theta$ can be calculated as $\Theta \leq 2 \pi / N_{p}$ and in this experiment is equal to $\Theta<0.45^{\circ}$, thus we chose $\Theta=0.4^{\circ}$ and the number of projections equal to 900 . The CCD chip was cooled down to $-20{ }^{\circ} \mathrm{C}$ to decrease its thermal, intrinsic noise. To obtain each shadowgram (projection), 5 EUV pulses were acquired using the CCD camera, in order to minimize a shot-to-shot variation in EUV pulse energy and to statistically average the gas transmission in each shadowgram, which in turn is beneficial for the accuracy of further 3-D reconstruction.

\section{EUV tomography results}

The tomography of the target has been performed for argon backing pressure of 1.5 bar. The EUV shadowgrams for $\Theta=0^{\circ}$ and $\Theta=90^{\circ}$ are depicted in Fig. 3a, c. Shadowgram in Fig. 3a shows that characteristic gas jets are produced as a result of free expansion of gas from the orifices into a vacuum. Such gas jets are typical for sonic expansion of gas flowing through a small orifice (sonic nozzle) under high pressure [4].

If argon is supplied to nozzles surrounded by similar nozzles, secondary jets are forming as a result of collision between two jets originating from the nozzles-primary jets. For larger distances $\sim 2.7 \mathrm{~mm}$ from the nozzle, one can observe formation of the tertiary jets as a result of collision between the secondary jets. The number of the secondary jets is at one smaller than the number of the primary jets. This mode of operation is similar to the data presented in [14], where only one kind of gas was supplied to all nozzles. Detailed study of gas backing pressure dependence on the target formation can also be found elsewhere $[14,15]$.

From the shadowgrams in Fig. 3a, c, it was possible to obtain the density of the target. The gas density maps of the 3-D gas density distributions were calculated from the EUV shadowgrams using the Eq. (2):

$\rho(x, y)=\frac{-\ln [\operatorname{Tr}(x, y)]}{\mu_{a} \cdot d(y)} \cdot m_{a t}$

where $\operatorname{Tr}(x, y)$ is a 2-D transmission map of the gas puff target, $\mu_{\mathrm{a}}=2 r_{0} \cdot \lambda \cdot f_{2}$ is an atomic photoabsorption cross section, $r_{0}=2.82 \times 10^{-15} \mathrm{~m}$ is the classical electron radius, $\lambda=13.5 \mathrm{~nm}$ is the wavelength, $f_{2}$ is the imaginary part of the atomic scattering factor, $d(y)$ is the path-length on which the EUV beam is absorbed in the gas, measured in direction of the EUV beam, horizontally, as indicated in Fig. $3 \mathrm{c}, m_{\mathrm{at}}$ is the atomic mass of the gas puff target material and $(x, y)$ are spatial coordinates at the detector plane, horizontal and vertical, respectively. The 1-D pathlength $d(y)$ can be found by measuring averaged FWHM widths of the density sections, obtained at various distances from the nozzle oriented as in Fig. 3c. The FWHM widths, corresponding to the path-lengths, are then interpolated to find analytical equations, $d(y)$, describing the path-length
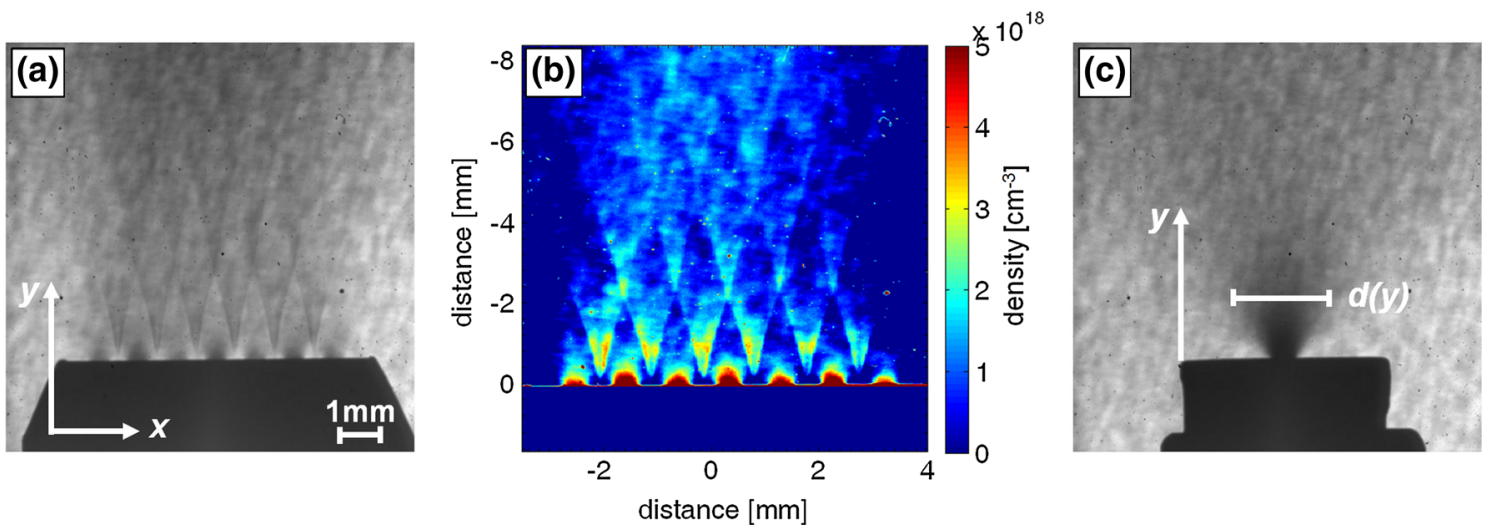

Fig. 3 EUV shadowgrams (raw data) of multi-jet gas puff target produced by supplying $\mathrm{Ar}$ gas to the nozzles at 1.5 bar backing pressure, for variable rotation angle $\Theta=0^{\circ}$ and $90^{\circ}$ (a and c,

respectively). 2-D density map (b) obtained from EUV shadowgram (a) using data from image (c) to obtain path-length $d(y)$ for density calculations 

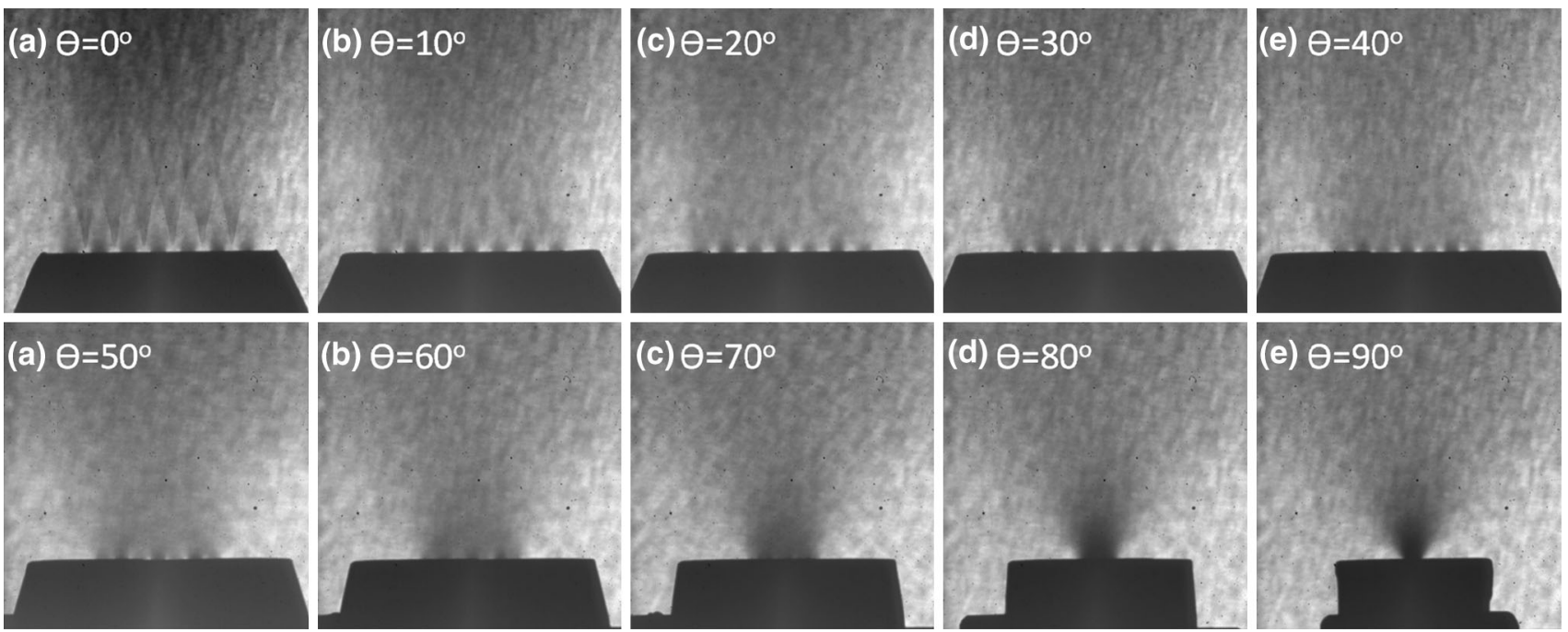

Fig. 4 Sequence of a few EUV shadowgram images of the target over $\pi / 2$ rotation angle

as a function of a distance from the nozzle plane $y$. It needs to be stressed that this method is greatly simplified, thus not highly accurate, however, allows to find the 2-D density maps for 3-D density distributions, assuming a uniform path-length $d(y)$ in orthogonal direction to the $\mathrm{x}-\mathrm{y}$ imaging plane. For the density calculations, we also assumed a monochromatic radiation; however, due to a certain bandwidth of the EUV radiation, equal to $\sim 1 \mathrm{~nm}$, measured as a FWHM of the spectrum depicted in Fig. 2b, there is additionally an error associated with slightly different absorption of the EUV radiation at different wavelengths. The 2-D density map obtained from shadowgram data is presented in Fig. 3b. The modulation depth of the gas density profiles in this case reaches $70 \%, 1.2 \mathrm{~mm}$ away from the nozzle plane, with maximum and minimum attainable densities of $\mathrm{Ar}$ equal to $2.3 \times 10^{18}$ and $6.6 \times 10^{17}$ atoms $/ \mathrm{cm}^{3}$, respectively, in the region where the secondary jets are forming.

Density resolution was also assessed as the smallest density change which this setup is capable of detecting. This can be estimated directly from Fig $3 b$ - a density map, for higher-order jets, where we can see primary jets very well ( 7 orifices above the nozzle plane reaching $5 \times 10^{18}$ atoms $\left./ \mathrm{cm}^{3}\right)$, then secondary jets $\left(2-3 \times 10^{18}\right.$ atoms $\left./ \mathrm{cm}^{3}\right)$ and tertiary jets $\left(1-1.5 \times 10^{18}\right.$ atoms $\left./ \mathrm{cm}^{3}\right)$ up to a distance of $-4 \mathrm{~mm}$ on vertical axis. Fourth-order jets are much more difficult to see; however, three of them (of four) are visible at $\sim-6 \mathrm{~mm}$ distance (reaching density $<1 \times 10^{18}$ atoms $/ \mathrm{cm}^{3}$ ). There is also some gas, surrounding fourth-order jets, with density down to $3 \times 10^{17}$ atoms $/ \mathrm{cm}^{3}$ and, in our opinion, it is on the edge of detecting capability for our setup.

Using a full data set of 900 radiography projections with additional 50 shadowgrams without the gas target-flat- field images, we performed a tomographic reconstruction of the gas puff target. The flat-field images (the valve was closed during shadowgram acquisition) were obtained before and after data set acquisition. Figure 4 shows a sequence of a few EUV shadowgram images of the target recorded over $\pi / 2$ rotation angle.

The standard reconstruction algorithm for parallel beam geometry in Octopus software [35] was used for processing of 2-D radiography projections into CT slices. VGStudio 2.1.5 software (http://www.volumegraphics.com) was used for stacking of $\mathrm{CT}$ slices and for the three-dimensional rendering and visualization. The slices were generated from projection data with a voxel size of $(25 \mu \mathrm{m})^{3}$. Some of those slices are presented in Fig. 5b-i for planes represented by dashed lines in Fig. 5a at various distances from the nozzle plane $y$. The dark numbers for each slice represent the slice number (1-512), starting from the very top of 2-D projections down. The white numbers were recalculated distances from the nozzle exit plane, based on the voxel size. The white areas represent regions with increased electron density, while the circular pattern present in all images is associated with the reconstruction artifacts. In Fig. 5b, only extruded part of the valve can be seen. Directly above the nozzle (Fig. 5c), the gas jets, formed by the nozzles (primary jets), are present. The number of gas jets corresponds to the number of the nozzles. As expected, the gas jets are circularly symmetric, even though it is not immediately evident from the data. Approximately $200 \mu \mathrm{m}$ away from the nozzles, secondary jets are formed in between the primary jets (Fig. 5d). Their number is at one less than the number of primary jets. From the top view, we can observe directly the cross section of those jets and precisely evaluate their shape which is impossible to see directly just from the 2-D projections. 

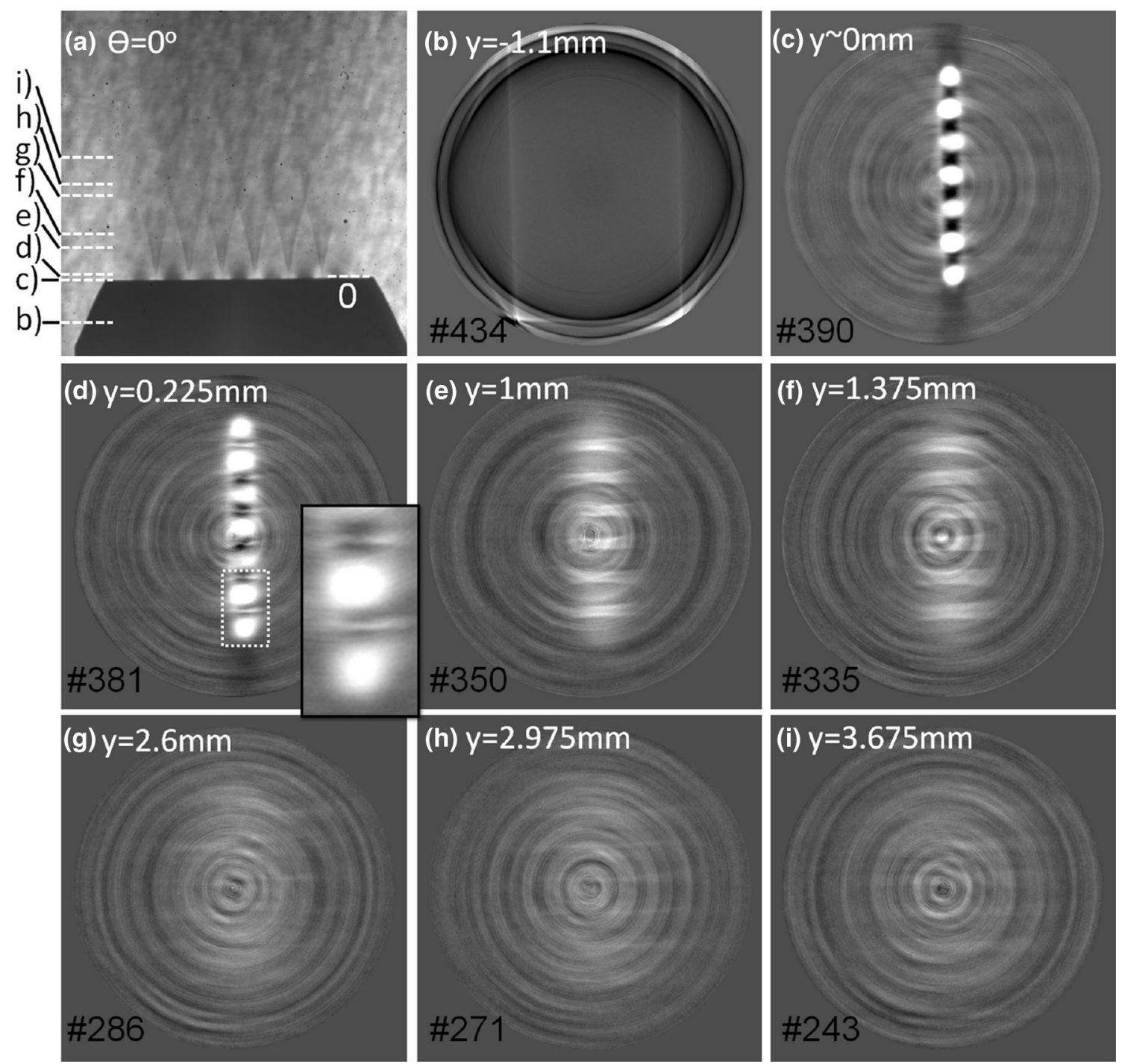

Fig. 5 Slices produced in the reconstruction process $(\mathbf{b}-\mathbf{i})$ for planes represented by dashed lines in (a) for various distances $y$ from the nozzle plane. The dark numbers are the slice number (1-512), starting from the very top of $2-\mathrm{D}$ projections down. The white numbers

Small inset in Fig. 5d shows the initial moment of formation of the secondary jets. If we are moving away from the nozzles, at the distance of $\sim 1 \mathrm{~mm}$, the main contribution to the reconstructed electron density is from the secondary jets, as can be seen in Fig. 5e. There is still, however, some gas density in between the secondary jets, which might be attributed to the primary jets. Moreover, we can also change the duty cycle of the modulated gaseous target by adjusting the distance from the nozzles. As can be observed in Fig. $5 \mathrm{f}$, at $\sim 1.4 \mathrm{~mm}$ away, the duty cycle for modulated target approaches $50 \%$, more than in Fig. 5e, which was $\sim 30 \%$. This is a very important feature that might be useful for HHG phase-matching to increase the conversion efficiency of the process. In the indicate distances from the nozzle exit plane. The white areas represent regions with increased electron density, while the circular pattern, present in all the images, is associated with the reconstruction artifacts

region, where secondary jets start to overlap (Fig. 5g), there is no density modulation, as presented in the corresponding slice; however, $\sim 400 \mu \mathrm{m}$ above that plane, we can again clearly observe some modulation associated with the formation of the tertiary jets as shown in Fig. 5h. Those jets, although less visible due to reduced density of $\sim 1.75 \times 10^{18}$ atoms $/ \mathrm{cm}^{3}(\sim 2.8 \mathrm{~mm}$ away from the nozzle plane), still change their width, and thus, the duty cycle for modulated density target can be optimized for efficient HHG in that region as well (Fig. 5i).

This is in accordance with the sinogram data. Sinogram is obtained as a set of projection vectors $\vec{x}$, obtained from 2-D shadowgrams at a particular distance from the nozzle $y$ versus the projection angle $\Theta$, which is depicted in Fig. 6a. The 

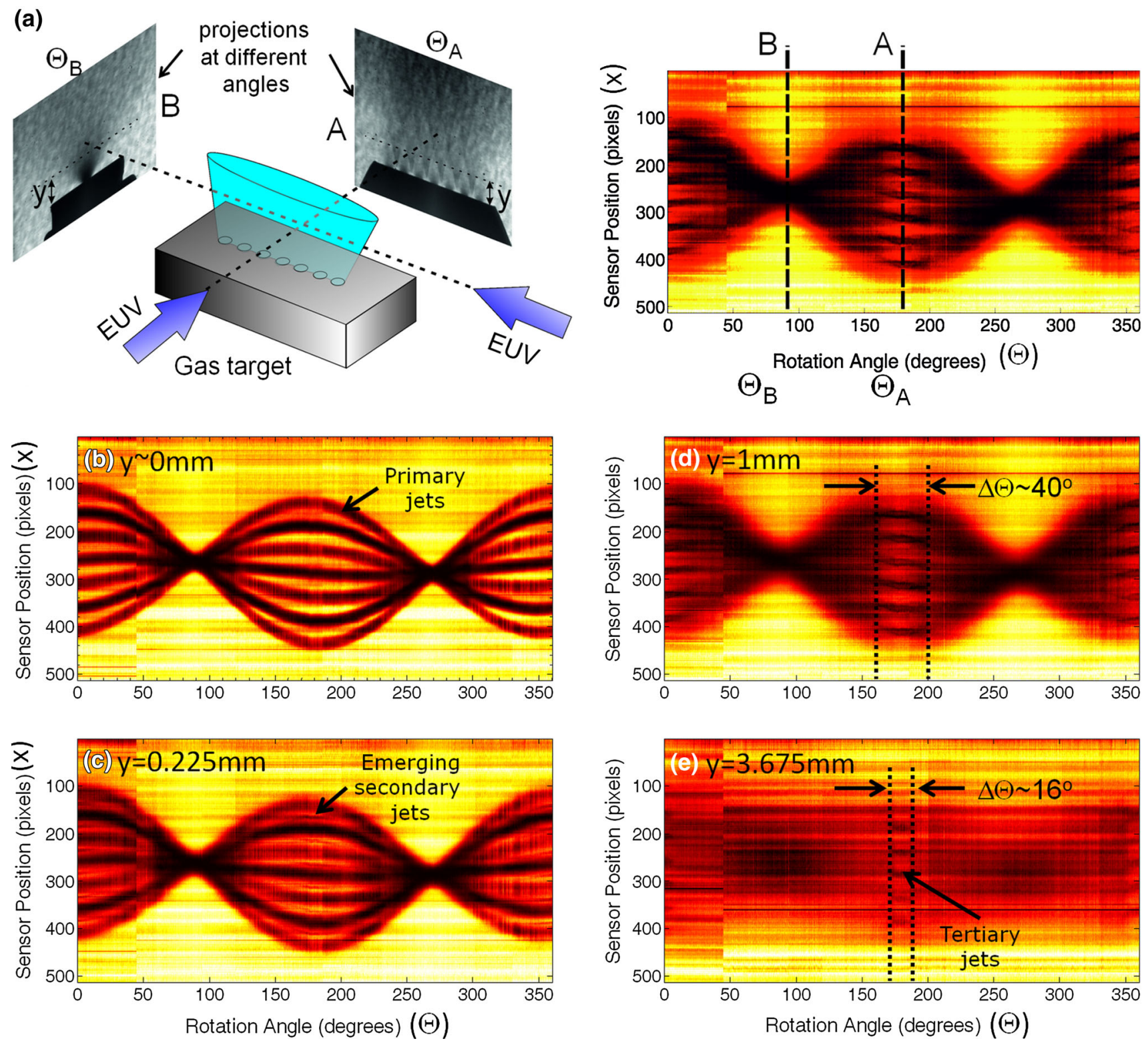

Fig. 6 Scheme for generating sinograms (a) and sinograms (b-e) obtained for various distances from the nozzle plane $y$ corresponding to slices presented in Fig. 5c-e, i)

sinograms for various reconstruction planes (slices) are presented in Fig. $6 \mathrm{~b}-\mathrm{e}$ and correspond directly to slices presented in Fig. 5c, d, e, i, because they were obtained at precisely the same distances from the nozzle plane $y$. Figure $6 \mathrm{~b}$ represents angular distribution of the projection data just above the nozzle, where only primary jets are present. Those jets are visible from $120^{\circ}$ to $240^{\circ}$ angles, over more than $60^{\circ}$ on each side of the central projection at $180^{\circ}$. $225 \mu \mathrm{m}$ above that plane, secondary jets start to emerge in between strong primary jets, which is depicted in Fig. 6c. At $1 \mathrm{~mm}$ above the nozzle plane (Fig. $6 \mathrm{~d}$ ), contribution of secondary jets is most significant; moreover, their angular spread is reduced to $\Delta \Theta \sim 40^{\circ}$. Weakly visible tertiary jets are also present in the sinogram data (Fig. 6e), with angular spread of $\Delta \Theta \sim 16^{\circ}$. Smaller angular spread means that their path-length $d(y)$ is much larger than of the other jets.

Modulation profiles were also obtained directly from slices and are presented in Fig. 7. Figure 7a corresponds to slice from Figs. 5 c, $7 \mathrm{~b}$ to $5 \mathrm{~d}, \ldots$, etc. The profiles in Fig. $7 \mathrm{a}, \mathrm{b}$ show very pronounced primary jets with saturated intensity in the reconstructed image. In Fig. 7b, small secondary jets appear, visible in the profile image, just in between strong primary jets. Those secondary jets are dominating the profile in Fig. $7 \mathrm{c}$, producing 
(a)
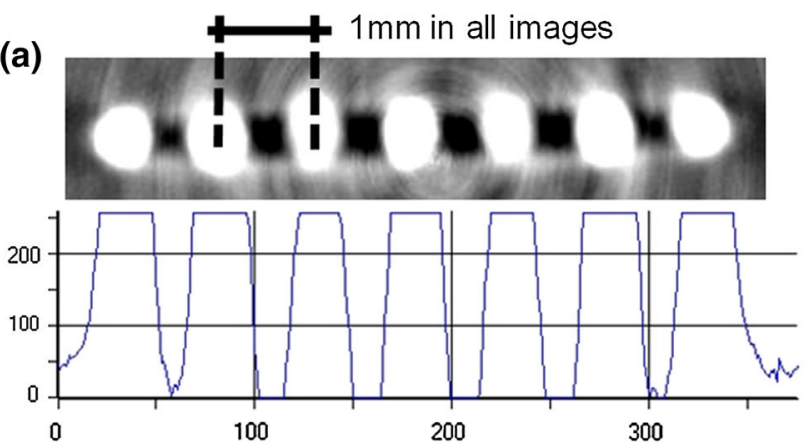

(b)

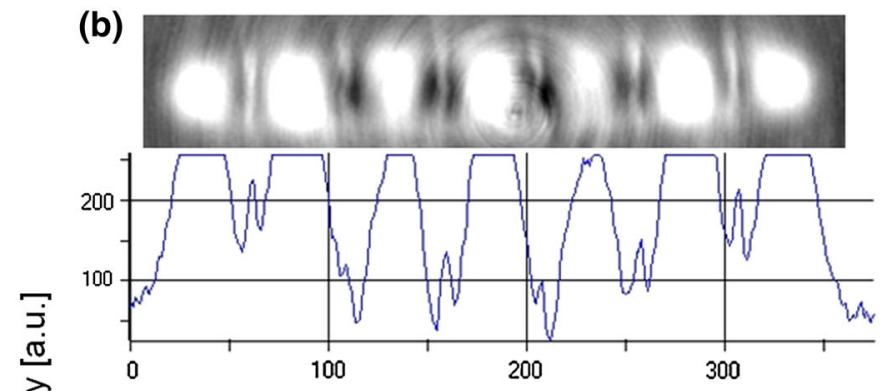

(c)

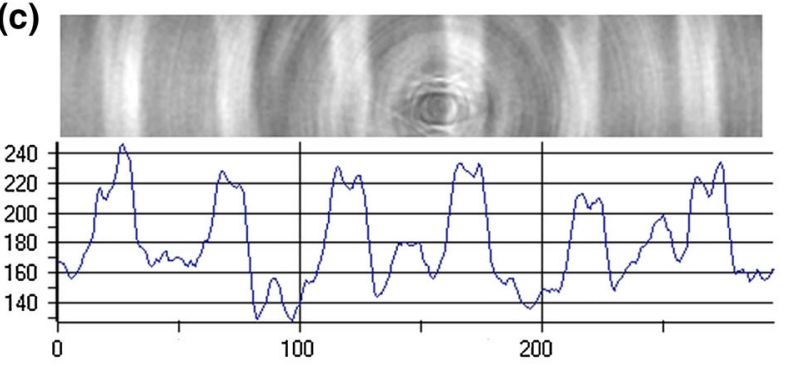

\section{(d)}
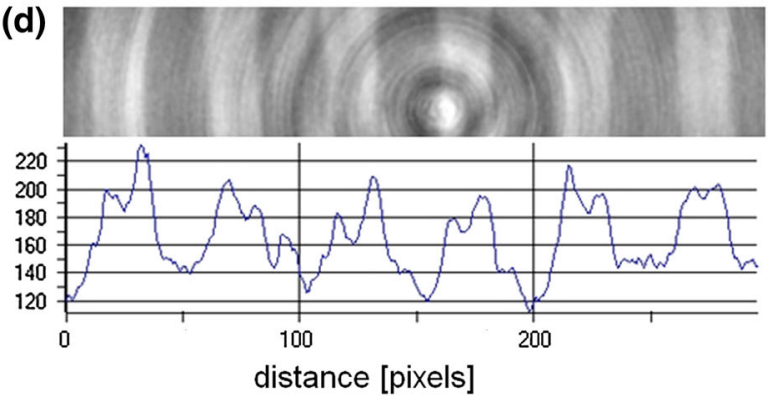

(e)
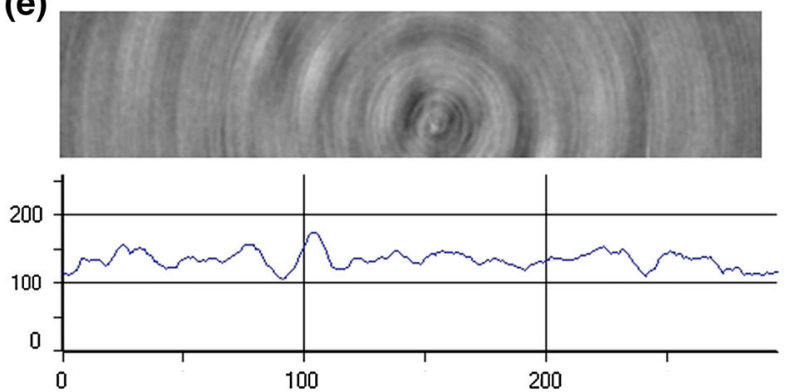

(f)
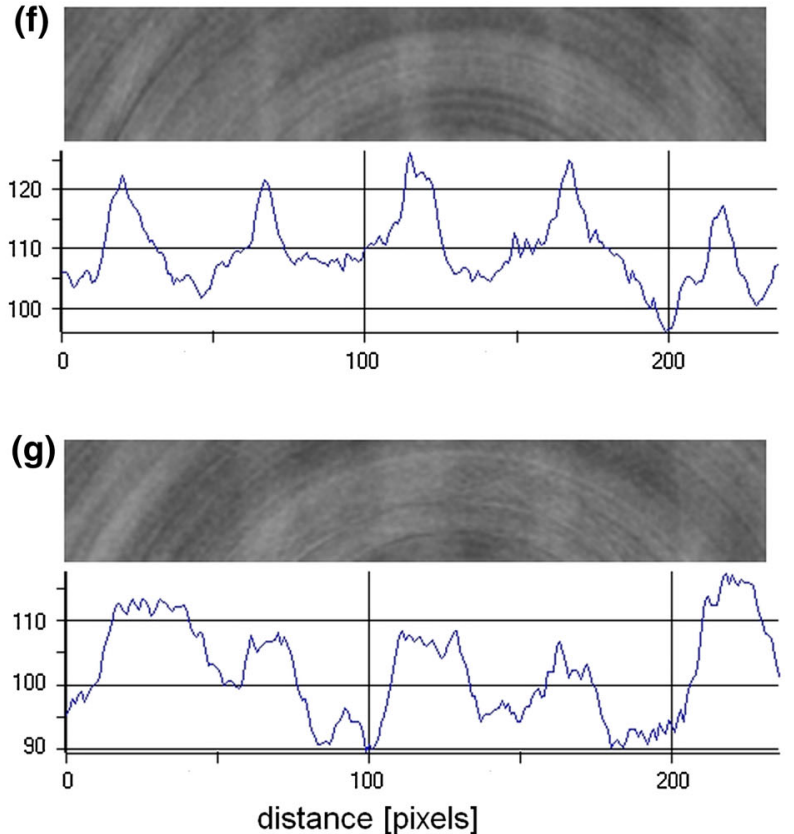

Fig. 7 Modulation profiles obtained directly from slices presented in Fig. 5, with 8 bits dynamic range of gray levels. Insets and profiles correspond to slices in Fig. 5

rectangular density profile with lower duty cycle. Figure $7 \mathrm{~d}$ depicts similar profile with higher duty cycle in the density modulation plot. Figure $7 \mathrm{e}$ shows no periodic structure in the density plot, due to previously explained collision of the secondary jets; however, Fig. 7f, g shows once again modulation originating from the formation of tertiary jets. Modulation depth of the density profile, formed by tertiary jets, is much smaller than in case of secondary jets (for data in Fig. $7 \mathrm{c}$ equal to $\sim 31 \%$ ) and is equal to $\sim 8 \%$ (Fig. $7 \mathrm{f}$ ) or $\sim 4 \%$ of the reconstruction dynamic range from 0 to 255 shades of gray. Combining the slices, we obtain a volumetric reconstruction of the multi-jet target. The result of 3-D tomographic reconstruction can be seen in Fig. 8, while the short movie of rotating 3-D target can be found in supplementary materials online. 


\section{Preliminary high-order harmonic generation measurements for modulated density gas puff targets}

Modulated density targets might be used in the future to enhance the conversion process in HHG. To achieve that a phase-matching between the laser fundamental beam and the harmonics, generated by coherent addition of HHG signal from a large collection of atoms, is required. It was theoretically demonstrated that HHG phase-matching in targets with modulated density will increase HHG signal, as reported in [20].

To prove that we have performed initial measurements of HHG signal from an array of seven nozzles, each $0.5 \mathrm{~mm}$ in diameter, arranged equidistantly in-line geometry over a distance of $9 \mathrm{~mm}(\sim 1.3 \mathrm{~mm}$ period $)$ and compared the emission to non-modulated target having the

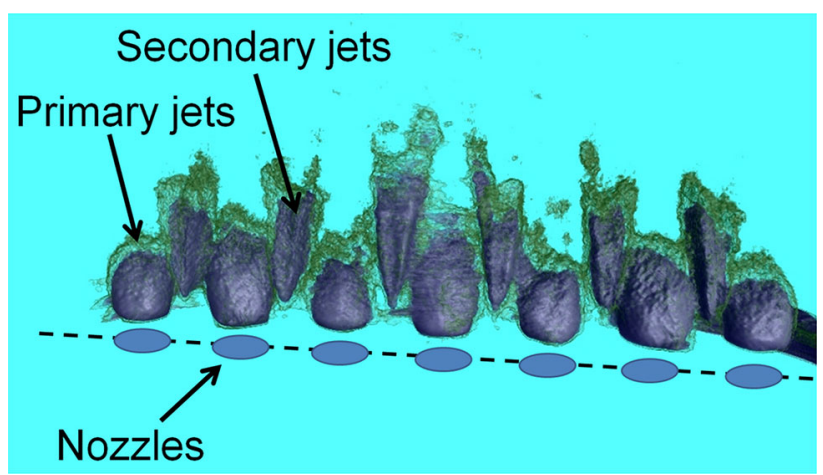

Fig. 8 Three-dimensional visualization of the reconstructed gas puff target showing primary and secondary gas jets. Additionally, positions of the nozzles were schematically indicated. Movie of rotating 3-D target can be found in supplementary materials online same length. The example result of spectral measurements for 21-31 harmonics is presented in Fig. 9, for argon gas backing pressure of $1 \mathrm{bar}, \lambda=810 \mathrm{~nm}$ wavelength, $t=40 \mathrm{fs}, E \sim 1 \mathrm{~mJ}$ pulses, focused by an $f=750 \mathrm{~mm}$ lens onto a targets $1.5 \mathrm{~mm}$ above the nozzle exit. The diameter of the focus at FWHM was $90 \mu \mathrm{m}$, giving the laser intensity in the focus of about $10^{14} \mathrm{~W} / \mathrm{cm}^{2}$. The modulated target density, measured using a shadowgraphy technique, was equal $(2.16 \pm 0.13) \times 10^{18}$ atoms $/ \mathrm{cm}^{3}$ across secondary gas jet and $(1.53 \pm 0.06) \times 10^{18}$ atoms/ $\mathrm{cm}^{3}$ between two jets, at the target density duty cycle equal to $37 \%$. Target density measured for the slit nozzles was equal to $(1.79 \pm 0.15) \times 10^{18}$ atoms $/ \mathrm{cm}^{3}$. Error quoted here is a \pm standard deviation from 20 measurements. The spectrum was measured using reflective diffraction grating $(1,200$ groves $/ \mathrm{mm})$ coupled to a back-illuminated CCD camera, sensitive to the EUV radiation (Andor, model DX440-BN). The increase in signal is modest at this time, $\sim 100 \%$ for most intense harmonics, due to the fact that the design of the nozzles was not particularly optimized for that purpose; however, it promises improvement in HHG efficiency and is a part of an ongoing work including simulations and experimental confirmation, which cannot be currently presented. Employing modulated density targets also allows tweaking the HHG emission spectrum, by channeling the energy flow to different harmonics, which was recently demonstrated in [36].

\section{Conclusions}

In summary, we have performed the tomographic reconstruction of the multi-jet gas puff target by combining shadowgraphy projection images into a 3-D reconstruction
Fig. 9 Spectral measurements for 9-mm-long modulated density gas puff target (dashed line) and for 9-mm-long slit nozzle with near-constant density (solid line)

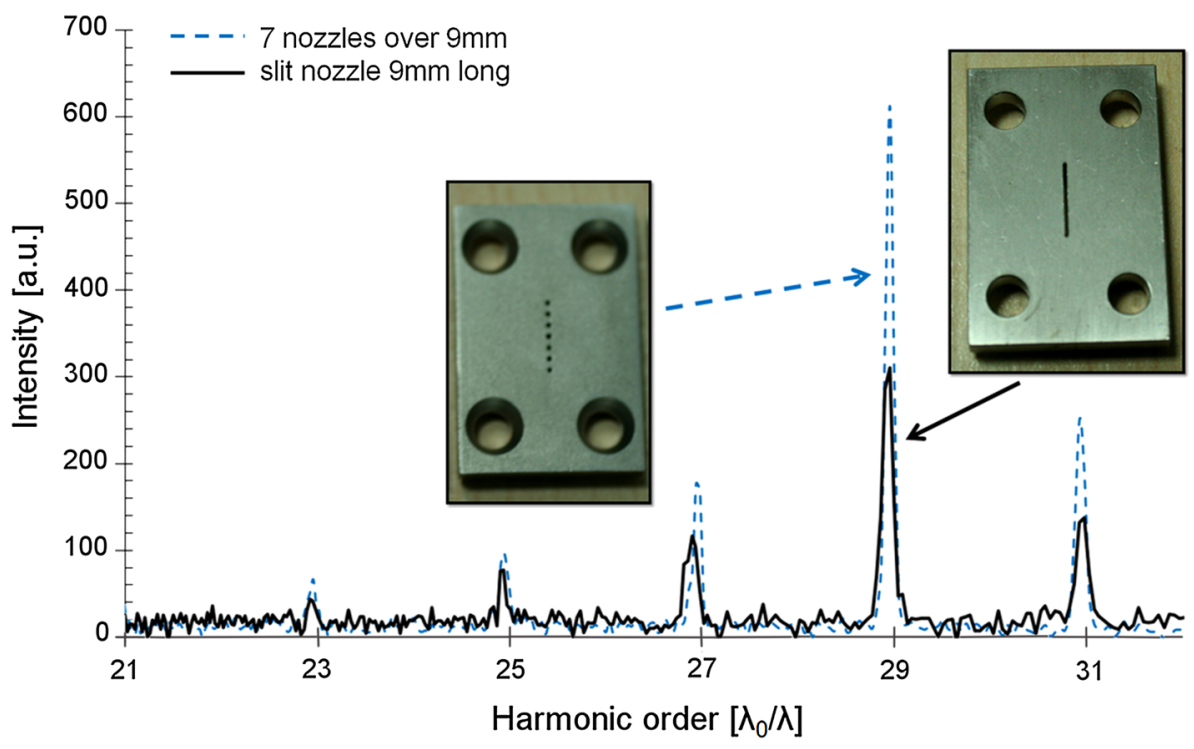


volume. Reconstructed gas jets compose a uniform, modulated density gas-comb-like pattern. The spatial density profiles along the nozzle array are strongly modulated. Their density and shape depend on the distance from the nozzle, which was experimentally studied in detail in this paper. Those results might be of high importance to optimize and characterize possible targets for phase-matched HHG and in turn possible future applications of improved efficiency of HHG sources. Some additional information regarding the secondary jets might be directly observed only form 3-D reconstructions. Even though the contrast of the shadowgram is not high, due to small gas backing pressure used in the experiment, the tomographic technique is capable of visualizing gas density distribution in the range of $10^{18}$ atoms per $\mathrm{cm}^{3}$ in much detail.

Acknowledgments The research was supported by the Ministry of Science and Higher Education of Poland and the European Commission's Seventh Framework Program (LASERLAB-EUROPEgrant agreement 284464 and COST Action MP0601 and MP1203) European Economic Area (EEA) grants. PWW greatly acknowledges the support from the National Centre for Science, award number DEC-2011/03/D/ST2/00296 and the National Centre for Research and Development, Lider programme, award \# LIDER/004/410/L-4/12/ NCBR/2013. ZZ and DK acknowledge support by Research and Development Centre for Advanced X-ray Technologies, ITMS code 26220220170, supported by the Research and Development Operational Programme, funded by the European Regional Development Fund and support of the Science and Technology Assistance Agency Bratislava (project No. APVV-0308-11).

Open Access This article is distributed under the terms of the Creative Commons Attribution License which permits any use, distribution, and reproduction in any medium, provided the original author(s) and the source are credited.

\section{References}

1. A. Pirri, C. Corsi, M. Bellini, Enhancing the yield of high-order harmonics with an array of gas jets. Phys. Rev. A 78, 011801(R) (2008)

2. A. Willner, F. Tavella, M. Yeung, T. Dzelzainis, C. Kamperidis, M. Bakarezos, D. Adams, M. Schulz, R. Riedel, M.C. Hoffmann, W. Hu, J. Rossbach, M. Drescher, N.A. Papadogiannis, M. Tatarakis, B. Dromey, M. Zepf, Coherent control of high harmonic generation via dual-gas multijet arrays. Phys. Rev. Lett. 107, 175002 (2011)

3. A. Behjat, G.J. Tallents, D. Neely, The characterization of a highdensity gas jet. J. Phys. D Appl. Phys. 30, 2872 (1997)

4. R.A. Smith, T. Ditmire, J.W.G. Tisch, Characterization of a cryogenically cooled high-pressure gas jet for laser/cluster interaction experiments. Rev. Sci. Instrum. 69, 3798 (1998)

5. T. Auguste, M. Bougeard, E. Caprin, P. D’Oliveira, P. Monot, Characterization of a high-density large scale pulsed gas jet for laser-gas interaction experiments. Rev. Sci. Instrum. 70, 2349 (1999)

6. R. Azambuja, M. Eloy, G. Figueira, D. Neely, Three-dimensional characterization of high-density non-cylindrical pulsed gas jets. J. Phys. D Appl. Phys. 32, L35 (1999)
7. V. Malka, C. Coulaud, J.P. Geindre, V. Lopez, Z. Najmudin, D. Neely, F. Amiranoff, Characterization of neutral density profile in a wide range of pressure of cylindrical pulsed gas jets. Rev. Sci. Instrum. 71, 2329 (2000)

8. N. Lemos, N. Lopes, J.M. Dias, F. Viola, Design and characterization of supersonic nozzles for wide focus laser-plasma interactions. Rev. Sci. Instrum. 80, 103301 (2009)

9. M. Landgraf, A. Schnell, M.C. Savert, C. Kaluza, Spielmann, high resolution 3D gas-jet characterization. Rev. Sci. Instrum. 82, 083106 (2011)

10. A.S. Boldarev, V.A. Gasilov, V.E. Levashov, K.N. Mednikov, A.S. Pirozhkov, M.S. Pirozhkova, E.N. Ragozin, Measurements of the xenon density in a pulsed jet from absorption of monochromatic soft Xrays. Quantum Electron. 34, 679 (2004)

11. D.L. Musinski, T.R. Pattinson, D.A. Steinman, R.B. Jacobs, Gas jet targets for laser plasma interaction studies. Plasma Phys. 24, 731 (1982)

12. Y.M. Li, R. Fedosejevs, Density measurements of a high-density pulsed gas jet for laser-plasma interaction studies. Meas. Sci. Technol. 5, 1197 (1994)

13. P.W. Wachulak, L. Wegrzynski, A. Bartnik, T. Fok, R. Jarocki, J. Kostecki, M. Szczurek, H. Fiedorowicz, Characterization of a dual-gas multi-jet gas puff target for high-order harmonic generation using EUV shadowgraphy. Laser Part. Beams 31(2), 219-227 (2013)

14. P.W. Wachulak, A. Bartnik, R. Jarocki, H. Fiedorowicz, Characterization of multi-jet gas puff targets for high-order harmonic generation using EUV shadowgraphy. Nucl. Instrum. Methods Phys. Res., Sect. B Beam Interact. Mater. At. 285(15), 102-106 (2012)

15. P.W. Wachulak, A. Bartnik, H. Fiedorowicz, R. Jarocki, J. Kostecki, M. Szczurek, Soft X-ray characterization of an elongated gas-puff target dedicated for laser-matter interaction experiments and high harmonic generation. Nucl. Instrum. Methods Phys. Res. B 276, 38 (2012)

16. A. Bartnik, V.M. Dyakin, I.Yu. Skobelev, A.Ya. Faenov, H. Fiedorowicz, M. Szczurek, Interaction of laser radiation with a dense gas target. Quantum Electron. 27(1), 68-71 (1997)

17. P.L. Shkolnikov, A. Lago, A.E. Kaplan, Optimal quasi-phasematching for high-order harmonic generation in gases and plasma. Phys. Rev. A 50, R4461 (1994)

18. M. Geissler, G. Tempea, T. Brabec, Phase-matched high-order harmonic generation in the nonadiabatic limit. Phys. Rev. A 62, 033817 (2000)

19. T. Auguste, B. Carré, P. Salières, Quasi-phase-matching of highorder harmonics using a modulated atomic density. Phys. Rev. A 76, 011802(R) (2007)

20. V. Tosa, V.S. Yakovlev, F. Krausz, Generation of tunable isolated attosecond pulses in multi-jet systems. New J. Phys. 10, 025016 (2008)

21. R. Spitzenpfeil, S. Eyring, C. Kern, C. Ott, J. Lohbreier, J. Henneberger, N. Franke, S. Jung, D. Walter, M. Weger, C. Winterfeldt, T. Pfeifer, C. Spielmann, Enhancing the brilliance of high-harmonic generation. Appl. Phys. A 96, 69-81 (2009)

22. A. Paul, R.A. Bartels, R. Tobey, H. Green, S. Weiman, I.P. Christov, M.M. Murnane, H.C. Kapteyn, S. Backus, Quasi-phasematched generation of coherent extreme-ultraviolet light. Nature 421, 51 (2003)

23. J. Seres, V.S. Yakovlev, E. Seres, Ch. Streli, P. Wobrauschek, Ch. Spielmann, F. Krausz, Coherent superposition of laser-driven soft-X-ray harmonics from successive sources. Nat. Phys. 3, 878 (2007)

24. J. Baruchel, J.Y. Buffiere, E. Maire, X-Ray Tomography in Material Science, (Peix, Ed. Hermes Science, Paris, France 2000)

25. W. Bauer, F.T. Bessler, E. Zabler, R.B. Bergmann, Computer tomography for nondestructive testing in the automotive industry, 
in Proceedings of the SPIE 5535, Developments in X-Ray Tomography IV, 464 (2004), doi:10.1117/12.555578

26. H.P. Hiriyannaiah, X-ray computed tomography for medical imaging. IEEE Signal Process. Mag. 14(2), 42-59 (1997). doi:10. $1109 / 79.581370$

27. A. Momose, T. Takeda, Y. Itai, K. Hirano, Phase-contrast X-ray computed tomography for observing biological soft tissues. Nat. Med. 2, 473-475 (1996). doi:10.1038/nm0496-473

28. Z. Zápražný, D. Korytár, V. Áč, P. Konopka, J. Bielecki, Phase contrast imaging of lightweight objects using microfocus X-ray source and high resolution CCD camera. JINST 7, C03005 (2012)

29. S. Boden, U. Hampel, M. Bieberle, Measurement of gas hold-up distribution in a stirred chemical reactor using X-ray cone-beam computed tomography. Chem. Eng. J. 139, 351-362 (2008)

30. S.W. Wilkins, T.E. Gureyev, D. Gao, A. Pogany, A.W. Stevenson, Phase-contrast imaging using polychromatic hard X-rays. Nature 384, 335-338 (1996)

31. X. Wu, H. Liu, Phase-space formulation for phase-contrast X-ray imaging. Appl. Opt. 44, 5847-5854 (2005)

32. R. Rakowski, A. Bartnik, H. Fiedorowicz, F. De Gaufridy De Dortan, R. Jarocki, J. Kostecki, J. Mikołajczyk, L. Ryć, M. Szczurek, P. Wachulak, Characterization and optimization of the laser-produced plasma EUV source at $13.5 \mathrm{~nm}$ based on a double-stream Xe/He gas puff target. Appl. Phys. B 101(4), 773-789 (2010)

33. R. Rakowski, A. Bartnik, H. Fiedorowicz, R. Jarocki, J. Kostecki, J. Mikołajczyk, A. Szczurek, M. Szczurek, I. Földes, Z. Tóth, Pulsed X-ray radiography of a gas jet target for laser-matter interaction experiments with the use of a CCD detector. Nucl. Instrum. Methods Phys. Res. Sect. A Accel. Spectrom. Detect. Assoc. Equip. 551, 139 (2005)

34. H. Fiedorowicz, A. Bartnik, R. Jarocki, J. Kostecki, J. Krzywiński, J. Mikołajczyk, R. Rakowski, A. Szczurek, M. Szczurek, Compact laser plasma EUV source based on a gas puff target for metrology applications. J. Alloy. Compd. 401, 99 (2005)

35. J. Vlassenbroeck, M. Dierick, B. Masschaele, V. Cnudde, L. Van Hoorebeke, P. Jacobs, Software tools for quantification of X-ray microtomography at the UGCT. Nucl. Instrum. Methods Phys. Res. Sect. A 580, 442-445 (2007)

36. T. Fok, Ł. Węgrzyński, M. Kozlova, J. Nejdl, P.W. Wachulak, R. Jarocki, A. Bartnik, H. Fiedorowicz, High-order harmonic generation using a multi-jet gas puff target: photonics Letters of Poland, accepted for publication, (2014)

37. CXRO X-Ray Interactions With Matter. http://henke.lbl.gov/ optical_constants/ 